\title{
Identification of circulating biomarkers in sera of Plasmodium knowlesi-infected malaria patients - comparison against Plasmodium vivax infection
}

\author{
Yeng Chen ${ }^{1,2^{*}}$, Choon K Chan ${ }^{3}$, Jesinda P Kerishnan ${ }^{1}$, Yee L Lau ${ }^{4}$, Yin-Ling Wong ${ }^{1}$ and Subash CB Gopinath ${ }^{1,5^{*}}$
}

\begin{abstract}
Background: Plasmodium knowlesi was identified as the fifth major malaria parasite in humans. It presents severe clinical symptoms and leads to mortality as a result of hyperparasitemia in a short period of time. This study aimed to improve the current understanding of P. knowlesi and identify potential biomarkers for knowlesi malaria.

Methods: In the present study, we have employed two-dimensional gel electrophoresis-coupled immunoblotting techniques and mass spectrometry to identify novel circulating markers in sera from $P$. knowlesi-infected patients. Specifically, we have compared serum protein profiles from $P$. knowlesi-infected patients against those of healthy or P. vivax-infected individuals.

Results: We identified several immunoreactive proteins in malarial-infected subjects, including alpha-2-HS glycoprotein (AHSG), serotransferrin (TF), complement C3C (C3), hemopexin (HPX), zinc-2-alpha glycoprotein (ZAG1), apolipoprotein A1 (Apo-A1), haptoglobin (HAP), and alpha-1-B-glycoprotein (A1BG). However, only TF and HPX displayed enhanced antigenicity and specificity, suggesting that they might represent valid markers for detecting $P$. knowlesi infection. Additionally, six P. knowlesi-specific antigens were identified (K15, K16, K28, K29, K30, and K38). Moreover, although HAP antigenicity was observed during $P$. vivax infection, it was undetectable in $P$. knowlesi-infected subjects.
\end{abstract}

Conclusions: We have demonstrated the application of immunoproteomics approach to identify potential candidate biomarkers for knowlesi malaria infection.

Keywords: Plasmodium knowlesi, Plasmodium vivax, Malaria, Immunoproteomics, Antigenicity

\section{Background}

Malaria has been known to be caused by major human malaria parasites - Plasmodium falciparum, $P$. vivax, $P$. malariae and P. ovale. P. knowlesi which was initially found in only long-tailed (Macaca fascicularis) and pigtailed (Macaca nemestrina) macaques [1]. It has been recognized as the fifth species of Plasmodium that causes malaria infection in humans [2,3]. Several cases of $P$. knowlesi infection in humans were reported in Malaysian Borneo, Myanmar, Philippines, Singapore, and Thailand [1,4], supporting the notion that P. knowlesi represents a major cause of malaria in Southeast Asia. $P$.

\footnotetext{
*Correspondence: chenyeng@um.edu.my; subash@unimap.edu.my 'Department of Oral Biology \& Biomedical Sciences, Faculty of Dentistry, University of Malaya, 50603 Kuala Lumpur, Malaysia

Full list of author information is available at the end of the article
}

knowlesi is the only malaria species that has a 24-hour asexual reproduction cycle (quotidian). Therefore, patients infected with $P$. knowlesi can easily reach lethal parasite densities in a relatively short period of time [5]. Respiratory distress, renal dysfunction, jaundice, hypoglycaemia and severe anaemia are the general clinical manifestations of severe malaria infection [6]. In addition, the severity of knowlesi malaria was found to be associated with hyperparasitemia and this has been reported in Malaysia and in other Southeast Asia regions $[5,6]$.

Like other malaria species, identification of $P$. knowlesi infection is achieved through examination of thick and thin blood films followed by Giemsa microscopy. This method uses specific morphological characteristics to differentiate parasites. However, accurate diagnosis of $P$. knowlesi by microscopy is often limited by the fact that P. knowlesi bares strong morphological resemblance to 
P. falciparum (early trophozoite stage) and $P$. malariae (erythrocytic stages) [7]. Thus, diagnosis of P. knowlesi usually requires molecular detection methods, which are performed in reference laboratories. In this regard, polymerase chain reaction (PCR) and molecular characterization currently represent the most reliable detection methods for P. knowlesi infection. Nevertheless, PCR-based techniques are not suitable for routine identification, since this method requires parasite DNA and is time consuming. In addition, $P$. knowlesi is frequently misdiagnosed as $P$. malariae through PCR and conventional microscopy [5]. Currently, there are no commercially available malaria rapid diagnostic tests are designed specifically for P. knowlesi detection. Although Plasmodium lactate dehydrogenase (pLDH) assay is more reliable in detecting $P$. knowlesi infection, the crossreactivity of $P$. knowlesi with $P$. falciparum-specific and $P$. vivax-specific pLDH has been shown [8]. Therefore, as stated by Cox-Singh et al. [5], there is currently a fundamental need for effective and practical diagnostic methods, which will not only contribute to reduced malariaassociated complications and mortality, but also facilitate global malaria control.

Notably, host biomarkers can be used to assess the risk of infection, examine protection against active diseases, or to determine therapeutic responses. Indeed, employing gene profiling, Schaecher et al. [9] has identified host biomarkers that can differentiate between lethal and non-lethal blood stages of murine malaria. Serum samples collected during the course of infection were utilized to analyze differential protein expression patterns, which were found to correlate with the degree of infection. Such data can provide insight into the cell regulatory mechanisms that participate in pathogenesis, immune responses and host recovery. Moreover, the identified proteins could be measured by serological tests to detect variants of malaria parasites while conducting epidemiological studies or implementing control programs. For example, serum angioprotein I and angioprotein 2/L have been suggested as diagnostic and prognostic biomarkers as well as potential therapeutic targets in cerebral malaria [10]. In the present study, we have examined the proteomic profiles of serum from $P$. knowlesi-infected patients to identify distinctive immunological protein features. Specifically, we have compared serum protein profiles from $P$. knowlesi-infected patients against those of healthy or $P$. vivax-infected subjects. Our findings have the potential to enhance our understanding of $P$. knowlesi and might contribute to the development of novel diagnostic approaches.

\section{Methods}

\section{Clinical samples}

From 200 serum samples previously screened for malaria parasites [11], we selected 15 samples for the current study. Notably, these samples corresponded to patients who were newly diagnosed with either P. knowlesi ( $\mathrm{n}=9$, parasitemia range: $0.04-22.80 \%$, age range: $29-55$ years, Male, Malaysian) or $P$. vivax $(\mathrm{n}=6$, parasitemia range: $0.10-0.50 \%$, age range: $29-50$ years, Male, nonMalaysian Asian). Additionally, 23 serum samples were collected from normal healthy individuals and were used as a control group (age range: $29-50$ years, Male, Malaysian). All samples were obtained with patients' written consent, and this study was approved by the University of Malaya Medical Centre Ethical Committee in accordance with $\mathrm{ICH}-\mathrm{GCP}$ guidelines for good clinical practice and the Declaration of Helsinki (PPUM/MDU/ 300/04/03).

\section{Two-dimensional electrophoresis (2-DE)}

Two-dimensional electrophoresis (2-DE) was performed as previously described by Chen et al. [12]. Briefly, $10 \mu \mathrm{l}$ of unfractionated whole human serum (either individual serum or pooled sera) was subjected to isoelectric focusing using $13-\mathrm{cm}$ rehydrated precast immobilized dry strips (pH 4-7) (GE Healthcare Bio-Sciences, Uppsala, Sweden). For the second dimension, focused sample within the strips was subjected to electrophoresis using an $8-18 \%$ gradient polyacrylamide gel in the presence of sodium dodecyl sulphate (SDS). All samples were analyzed in duplicate. The 2-DE gels were silver stained as described by Heukeshoven and Dernick [13]. For mass spectrometric analysis, gels were stained using Coomassie Brilliant Blue (CBB) or a modified mass spectrometry (MS) silver staining method, as described by Shenvchenko et al. [14]. CBB gel plugs were used instead of silverstained gel plugs when a higher peptide concentration was expected from in-gel digestion.

\section{Mass spectrometry analysis and database search}

Selected spots were excised and subjected to in-gel tryptic digestion using the commercially available Proteo Extract $^{\mathrm{tm}}$ All-in-One Trypsin Digestion Kit (Calbiochem, Darmstadt, Germany). Mass spectrometry (MS) analysis was performed at the Faculty of Biological Sciences Proteomic Centre, National University of Singapore. After digestion, the resulting peptides were mixed with CHCA matrix solution $(5 \mathrm{mg} / \mathrm{ml}$ of cyano-4-hydroxycinamic acid in $0.1 \%$ trifluoroacetic acid [TFA] and 50\% acetonitrile $[\mathrm{ACN}])$ in 1:2 ratio and spotted onto a matrixassisted laser desorption/ionization (MALDI) target plate. Peptide mass spectra were obtained using an ABI 4800 Proteomics Analyzer MALDI-TOF/TOF Mass Spectrometer (Applied Biosystems, Foster City, CA, USA). For MS analysis, 1,000 shots were accumulated for each sample. MS data were automatically obtained with the five most intense ions for MS/MS. Peptides were subsequently subjected to MS/MS analyses using air with collision energy of $2 \mathrm{kV}$ and a collision gas pressure of $\sim 1 \times 10^{-6}$ Torr. The 
stop conditions were set to accumulate approximately 2,000 to 3,000 shots, depending on the quality of the spectra. The Mascot search engine (version 2.1; Matrix Science, London, UK) was used to analyze all of the tandem MS results. Also, GPS Explorer ${ }^{\text {Ts }}$ software (version 3.6; Applied Biosystems) was employed in combination with the Mascot search engine for peptide identification. The search parameters allowed for $\mathrm{N}$ terminal acetylation, $\mathrm{C}$-terminal cysteine carbamidomethylation (fixed modification), and methionine oxidation (variable modification). The peptide and fragment mass tolerance were set to $100 \mathrm{ppm}$ and $\pm 0.2 \mathrm{Da}$, respectively. Moreover, peptide mass fingerprinting (PMF) parameters for the data search were as follows: one missed cleavage allowed in trypsin digest; monoisotropic mass value; \pm 0.1 Da peptide mass tolerance; and $1+$ peptide charge state. Initial protein identification was determined by comparing peptide masses to a database of tryptic peptides from known proteins (ProteinPilot proteomics software [4800] Proteomic Analyzer; Applied Biosystems), and a score was assigned based on similarity to theoretically and experimentally determined masses. Analyses were conducted using International Protein Index (http://www. ebi.ac.uk/IPI/), NCBI, Unigene (version 3.38), and PlasmoDB (version 8.0; http://plasmodb.org/plasmo/) databases for human proteomics. There were a total of 10,719 entries included in the database search. Search scores of $>82$ (Mascot NCBI database) or $>30$ (Mascot search engine using PlasmoDB) were considered as significant.

\section{Immunoblotting}

For immunoblotting, we analyzed $10 \mu \mathrm{l}$ of pooled, unfractionated human serum. Following electrophoresis, the 2-DE gels for the pooled serum samples were grouped into five categories: (a) normal pooled sera probed with normal pooled sera, (b) normal pooled sera probed with P. knowlesi pooled sera, (c) P. knowlesi pooled sera probed with normal pooled sera, (d) $P$. knowlesi pooled sera probed with $P$. knowlesi pooled sera, (e) $P$. knowlesi pooled sera probed with $P$. vivax pooled sera. Each of the gels was transferred onto nitrocellulose membrane using the Multiphor II Novablot semi-dry system (GE Healthcare, Sweden). The blotted nitrocellulose membranes were then blocked with SuperBlock (Pierce, USA) and washed three times with Tris-buffered saline-Tween 20 (TBST). The membranes were subsequently incubated overnight $\left(4^{\circ} \mathrm{C}\right)$ with the indicated primary antibodies, which corresponded to $P$. knowlesi infection, $P$. vivax infection, or normal healthy controls (all diluted at 1:50). After another washing step, the membranes were incubated with monoclonal antihuman Immunoglobulin M (IgM) conjugated to horseradish peroxidase (HRP) (Invitrogen, USA) at a dilution of 1:6,000 for $1 \mathrm{~h}$ at room temperature. The resulting immunocomplexes were visualized using chemiluminescent blotting reagent (Pierce, USA) and X-ray film $(18 \times 24 \mathrm{~cm}$; Kodak).

\section{Differential image and data analysis}

We utilized LabScan image scanner (version 5; Amersham, Germany) to capture and store the 2-DE gel images. PD-Quest ${ }^{\text {tix }}$ 2-D gel analysis software (Version 8.0.1, Bio-Rad) was used to detect, match, and quantify the distinct protein spots. In order to identify differentially expressed serum proteins, the percentages of volume contributions (vol\%) were calculated (i.e., the spot volume of a specific protein as a percentage of the total spot volume of all proteins in the gel, including unresolved peptides).

\section{Statistical analysis}

Protein levels in the gels are presented as mean vol\% \pm SD (standard deviation). The variance ratio test (F) was used to analyse differences between control subjects and patients. Correlations between the variables were examined using GraphPad Prism 5 software. A p-value of less than 0.05 was considered as statistically significant.

\section{Functional enrichment and protein interaction analysis}

Functional enrichment and protein interactions were analysed using web-based bioinformatics tools. DAVID v6.7 (Database for Annotation, Visualization and Integrated Discovery) was employed for protein functional enrichment analysis $[15,16]$. DAVID bioinformatics provide a comprehensive biological knowledgebase of functional annotation tools for understanding the biological meaning behind large lists of genes or proteins. The functional categorization is considered significant when the p-value is less than 0.05 . The identified host-specific proteins were further evaluated using STRING v9.1 (Search Tool for the Retrieval of Interacting Genes), which is an application that aggregates available databases of known and predicted protein-protein associations [17].

\section{Results}

\section{Differential expression of antigenic proteins}

We have performed a 2-DE assay that allowed for rapid detection and differentiation of $P$. knowlesi from other Plasmodium species. We first separated unfractionated normal control sera samples with 2-DE and observed high-resolution profiles, which were made up of several distinct protein clusters (Figure 1a). Sera samples from $P$. knowlesi-infected patients, presumably containing $P$. knowlesi antigens, were also separated by 2-DE (Figure 1b). Subsequently, the 2-DE profiles from these patients were compared with those obtained from normal individuals, revealing key differences in the expression of several serum 


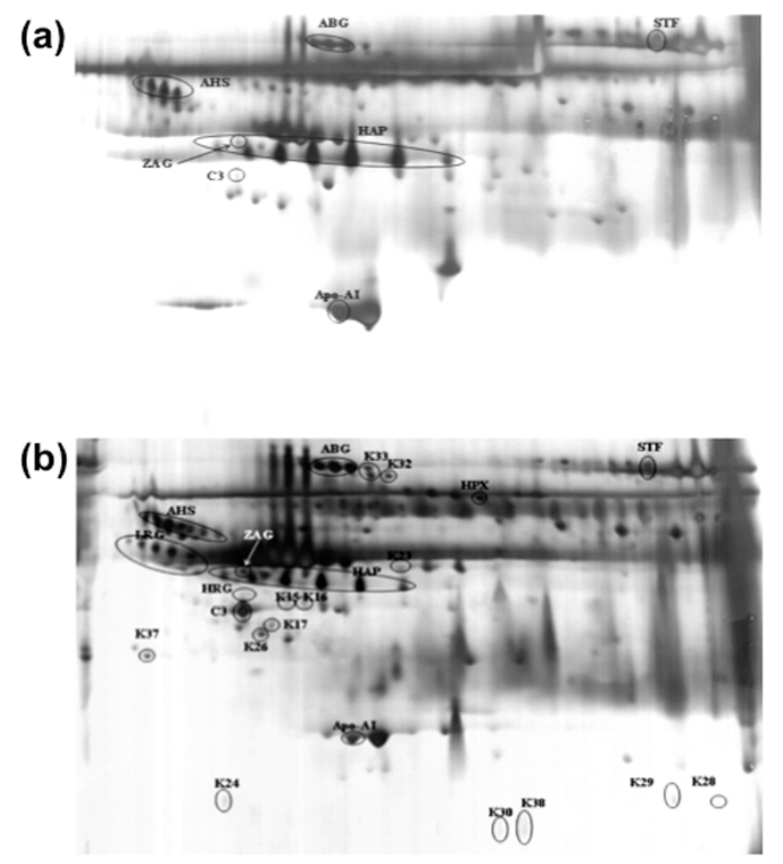

Figure 1 Representative 2-DE serum protein profiles for (a) control (b) P. knowlesi-infected individuals. Unfractionated serum samples from patients and controls were subjected to 2-DE and silver stained. Protein spots were compared and analysed using PD-Quest ${ }^{\text {TM }}$ 2-D gel analysis software (version 8.0.1, Bio-Rad).

proteins. Notably, knowlesi malaria patients displayed protein spots/clusters that appeared to undergo up- or downregulation (Figure $1 b$ ).

\section{Identification of expressed biomarkers by mass spectrometry}

We analysed the protein clusters using PD-Quest TM 2-DE gel analysis software (version 8.0.1, Bio-Rad), which indicated that there were a total of 200 and 124 protein spots detected by 2-DE for the P. knowlesi and normal samples, respectively. Therefore, 76 protein spots were differentially observed in malaria knowlesi sera compared to normal controls.

We further identified the following differentiallyexpressed host-specific proteins by MS: alpha-2-HS glycoprotein (AHSG), serotransferrin (TF), complement C3c (C3), hemopexin (HPX), zinc-2-alpha glycoprotein (ZAG1), apolipoprotein A1 (Apo-A1), haptoglobin (HAP), and alpha-1-B-glycoprotein (A1BG). All of the proteins were aberrantly expressed in patient samples as compared to normal controls. Indeed, as shown in the Table 1 , we observed significant increases in C3, ZAG1, HPX, AHS, and TF during knowlesi malaria infection (fold upregulation: $8.99,3.40,1.60,1.25$, and 1.15 , respectively; $\mathrm{p}<0.05)$. On the other hand, the expression of HAP, Apo-A1, and A1BG were significantly decreased (fold down-regulation: $0.42,0.34$, and $0.53 ; \mathrm{p}<0.05$ ) in knowlesi samples (Figure 1a-b). Additionally, we detected the following malaria-specific antigens: K15, K16, K17, K23, K26, K28, K29, K30, K32 K33, and K38 (Tables 1, 2, and 3).

The above results were confirmed through the use of MALDI-TOF/TOF and database searches. The PlasmoDB database was employed to blast MS/MS-identified peptides against the hypothetical $P$. knowlesi $\mathrm{H}$ strain proteome, which is only comprised of computationally predicted sequences [18]. This database contains the genomes of various mammalian Plasmodium species (P. falciparum, P. knowlesi, P. vivax, and P. yoelii), which range from 23 to $27 \mathrm{Mb}$ across 14 chromosomes and comprise approximately 5,500 genes (http://plasmodb.org/plasmo/). A remarkable $77 \%$ of these genes represent orthologous between these four species, and almost one-half of the genes encode conserved hypothetical proteins of unknown function. However, although PlasmoDB contains the most up-to-date annotation on $P$. knowlesi sequences, it is incomplete. Thus, it cannot be ruled out that some genes may be missing or incorrectly annotated in the PlasmoDB database. For this reason, experimental verification will be needed to assess whether current data offer a comprehensive view of the Plasmodium proteome, especially in the case of $P$. knowlesi. Nevertheless, our findings provide evidence to support the existence of some of these predicted sequences. The Mascot accession numbers, isoelectric points ( $\mathrm{pI}$ ), and molecular mass $(\mathrm{Mr})$ values associated with the identified proteins are listed in Tables 4 and 5.

\section{Detection of immunocomplexed biomarkers by 2-DE immunoblotting}

In order to confirm the 2-DE image analysis and MS results, we performed immunoblotting with pooled patient sera (anti-P. knowlesi antibodies). Specifically, our immunoblotting analysis involved five distinct conditions to allow

\section{Table 1 The relative expression of host specific proteins} among the sera of malaria patients

\begin{tabular}{ll}
\hline Host specific proteins & Fold changes \\
\hline 1. Alpha-2-HS glycoprotein (AHSG) & 1.28 \\
2. Serotransfererrin (TF) & 1.15 \\
3. Complement C3C (C3) & 8.99 \\
4. Hemopexin (HPX) & 1.60 \\
5. Zinc-2-alpha glycoprotein (ZAG1) & 3.40 \\
6. Haptoglobin (HAP) & 0.42 \\
7. Apolipoprotein A-I (Apo-A1) & 0.34 \\
8. Alpha-1-B-glycoprotein (A1BG) & 0.53
\end{tabular}

Fold change measures the degree of change in the protein of the $P$. knowlesiinfected individuals $(n=9)$, compared to normal controls $(n=23)$. This is measured by dividing the average spot intensity in the infected persons by the average spot intensity in the controls. 
direct comparison of $P$. knowlesi-infected sera against normal sera and sera from $P$. vivax-infected patients. The following conditions were tested (categories a-e): (a) normal pooled sera probed with normal pooled sera, (b) normal pooled sera probed with $P$. knowlesi pooled sera, (c) $P$. knowlesi pooled sera probed with normal pooled sera, (d) $P$. knowlesi pooled sera probed with $P$. knowlesi pooled sera, (e) $P$. knowlesi pooled sera probed with $P$. vivax pooled sera are displayed in Figures 2 and 3. Immunoblotting of 2-DE membranes revealed significant variations in the control (Figure 2a, b) and patient (Figures 2c, d and 3) sera profiles. In Figure 2, only categories 'a' (negative control) and 'c' (positive control) failed to show immunogenic spots. However, the remaining categories (Figures $2 \mathrm{~b}, \mathrm{~d}$, and 3) revealed many immunogenic host-specific and malaria antigens. Strikingly, we could specifically distinguish differences when immunoblotting vivax malaria patients with sera from knowlesi-infected patients.

Immunogenic host-specific proteins are displayed in Table 2. Some of these malaria serum-reactive proteins could be detected in both non-infected and infected serum, including AHSG, A1BG, and Apo-AI (Figure 2b, d). However, ZAG1 and C3 appeared prominently for both individual patients in categories $\mathrm{d}$ and e. In addition, the A1BG signal was observed in categories d and e. Notably, HAP was the only protein that showed immunogenicity in category 'e' but not in category ' $d$ '. TF was detected in category ' $d$ '. HPX only appeared in the category ' $d$ ' immunoblot, whereas HRG was exclusively observed in category ' $\mathrm{e}$ '.

With regard to malaria antigens, immunogenic spots for $\mathrm{K} 15, \mathrm{~K} 16, \mathrm{~K} 28, \mathrm{~K} 29, \mathrm{~K} 30$, and $\mathrm{K} 38$ were specific to $P$. knowlesi, as they could not be detected with antibodies from P. vivax-infected patients (Table 3 and Figure 3). However, only K15, K28, K30, and K38 were considered

Table 2 Host antigenic proteins show on the 2-DE immunoblotted nitrocellulose membrane

\begin{tabular}{|c|c|c|c|c|c|}
\hline \multirow{2}{*}{$\begin{array}{l}\text { Host } \\
\text { antigenic } \\
\text { proteins }\end{array}$} & \multicolumn{5}{|c|}{ Category } \\
\hline & (a) & (b) & (c) & (d) & (e) \\
\hline 1. AHSG & - & I & - & I & / \\
\hline 2. TF & - & - & - & / & - \\
\hline 3. C3 & - & - & - & / & / \\
\hline 4. HPX & - & - & - & / & - \\
\hline 5. ZAG1 & - & - & - & / & / \\
\hline 6. HAP & - & - & - & - & / \\
\hline 7. Apo-A1 & - & / & - & / & / \\
\hline 8. A1BG & - & I & - & / & / \\
\hline 9. $\mathrm{HRG}$ & - & - & - & - & / \\
\hline
\end{tabular}

/Proteins of the patients or normal pooled serum recognized by the primary antibody.

-Proteins of the patients or normal pooled serum not recognized by the primary antibody.
Table 3 Antigenic proteins show on the 2-DE immunoblotted nitrocellulose membrane

\begin{tabular}{llllll}
\hline $\begin{array}{l}\text { Antigenic } \\
\text { proteins }\end{array}$ & \multicolumn{2}{l}{ Category } & & & \\
\cline { 2 - 6 } & (a) & (b) & (c) & (d) & (e) \\
\hline 1. K15 & - & - & - & $(/)$ & - \\
2. K16 & - & - & - & $(/)$ & - \\
3. K17 & - & - & - & - & $/$ \\
4. K23 & - & - & - & - & $/$ \\
5. K26 & - & - & - & - & $/$ \\
6. K28 & - & - & - & $(/)$ & - \\
7. K29 & - & - & - & $(/)$ & - \\
8. K30 & - & - & - & $(/)$ & - \\
9. K32 & - & - & - & $/$ & $/$ \\
10. K33 & - & - & - & $/$ & $/$ \\
11. K38 & - & - & - & $(/)$ & - \\
\hline
\end{tabular}

(/)Antigens specifically detected in the knowlesi serum.

/Proteins of the patients or normal pooled serum recognized by the primary antibody.

-Proteins of the patients or normal pooled serum not recognized by the primary antibody.

to be significant (i.e., search score of $>30$ ). These four novel antigens were observed in 6 (66.7\%), 5 (55.6\%), 8 (95.1\%), and $9(100 \%)$ of the stained 2-DE gels, showing an average vol\% of $0.00638,0.00589,0.04782$, and 0.08956 (K15, K28, K30 and K38, respectively).

\section{Functional enrichment and protein interaction analysis}

In order to extend the above results, we employed DAVID v6.7 (http://david.abcc.ncifcrf.gov/) to perform functional ontology enrichment analyses (i.e., biological processes, molecular functions, cellular components, and pathways) for 11 significantly identified proteins (Table 6). This functional analysis revealed that AHSG, C3, and histidine-rich glycoprotein precursor (HRG) were involved in regulating responses to external stimuli, whereas HAP, HPX and TF participated in iron homeostasis. In addition, molecular function analysis showed that AHSG, C3 and HRG play a role in endopeptidase and peptidase inhibitor activity. Also, the majority of the proteins were located in the extracellular region. However, cellular components analysis revealed that Apo-A1, fibrinogen $\gamma$ chain (FGG), HRG, and TF could be found in secretory granules and membrane-bound vesicles. In addition, KEGG pathway analysis indicated significant participation of C3 and FGG in complement and coagulation cascades pathways $(\mathrm{p}=0.026957)$.

The STRING database (http://string-db.org/) is a curetted knowledge database that relies on evidence from highthroughput proteomic, genomic, and co-expression studies [19]. We performed protein interaction analysis using this database, which revealed an interaction network involving the identified host-specific proteins (AHSG, TF, C3, HPX, ZAG1, HAP, Apo-A1, HRG, A1BG, FGG and LRG1) 
Table 4 Mass spectrometric identification of host-specific protein spot clusters from serum protein profiles using MASCOT search engine and NCBI database

\begin{tabular}{|c|c|c|c|c|c|c|c|}
\hline Spot ID & $\begin{array}{l}\text { Mascot accession } \\
\text { number }\end{array}$ & pl & $\begin{array}{l}\text { Theoretical } \\
\text { mass }(\mathrm{Da}) / \mathrm{pl}\end{array}$ & $\begin{array}{l}\text { Sequence } \\
\text { coverage }\end{array}$ & $\begin{array}{l}\text { Search } \\
\text { score }\end{array}$ & $\begin{array}{l}\text { Queries } \\
\text { match }\end{array}$ & $\begin{array}{l}\text { Expected } \\
\text { value }\end{array}$ \\
\hline $\begin{array}{l}\text { 1. Chain C, Human Complement Component C3C } \\
\text { (C3) }\end{array}$ & gi|78101271 & 4.79 & 40217 & $10 \%$ & 218 & 6 & $1.8 \mathrm{e}^{-015}$ \\
\hline 2. Transferrin (TF) & gi|553788 & 6.00 & 55233 & $13 \%$ & 82 & 10 & 0.082 \\
\hline $\begin{array}{l}\text { 3. Chain A, Crystal Structure Of Lipid-Free Human } \\
\text { Apolipoprotein A-I (ApoA1) }\end{array}$ & gi|90108664 & 5.27 & 28061 & $62 \%$ & 357 & 26 & $2.3 \mathrm{e}^{-029}$ \\
\hline $\begin{array}{l}\text { 4. Leucine-rich } a_{2} \text {-glycoprotein precursor [Homo } \\
\text { sapiens] (LRG1) }\end{array}$ & gi|16418467 & 6.45 & 38382 & $31 \%$ & 358 & 16 & $1.8 \mathrm{e}^{-029}$ \\
\hline $\begin{array}{l}\text { 5. Hemopexin, isoform CRA_d [Homo sapiens] } \\
\text { (HPX) }\end{array}$ & gi|119589127 & 6.24 & 43781 & $13 \%$ & 112 & 8 & $7.3 e^{-005}$ \\
\hline 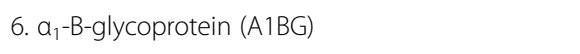 & gi|69990 & 5.65 & 52479 & $39 \%$ & 449 & 20 & $1.5 e^{-038}$ \\
\hline 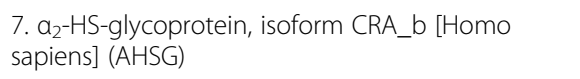 & gi|119598594 & 4.81 & 23148 & $6 \%$ & 121 & 2 & $9.2 e^{-006}$ \\
\hline 8. Haptoglobin [Homo sapiens] (HAP) & gi|3337390 & 6.14 & 38722 & $28 \%$ & 202 & 15 & $7.3 e^{-014}$ \\
\hline $\begin{array}{l}\text { 9. Histidine-rich glycoprotein precursor [Homo } \\
\text { sapiens] (HRG) }\end{array}$ & gi|4504489 & 7.09 & 60510 & $13 \%$ & 114 & 10 & $4.6 \mathrm{e}^{-005}$ \\
\hline 10. Chain B, Human Zinc- $a_{2}$-glycoprotein (ZAG1) & gi|4699583 & 5.70 & 31854 & $51 \%$ & 523 & 22 & $5.8 \mathrm{e}^{-046}$ \\
\hline 11. Fibrinogen gamma chain (FGG) & gi|930064 & 6.49 & 24337 & $45 \%$ & 56 & 10 & 33 \\
\hline
\end{tabular}

Table 5 Mass spectrometric identification of unknown protein spot clusters from serum protein profiles using MASCOT search engine, NCBI database and Plasmo DB

\begin{tabular}{|c|c|c|c|c|c|c|c|c|}
\hline Spot ID & $\begin{array}{l}\text { Mascot accession } \\
\text { number }\end{array}$ & $\begin{array}{l}\text { ID } \\
\text { Number }\end{array}$ & $\mathrm{pl}$ & $\begin{array}{l}\text { Theoretical } \\
\text { mass } \\
(\mathrm{Da}) / \mathrm{pl}\end{array}$ & $\begin{array}{l}\text { Sequence } \\
\text { coverage }\end{array}$ & $\begin{array}{l}\text { Search } \\
\text { score }\end{array}$ & $\begin{array}{l}\text { Queries } \\
\text { match }\end{array}$ & $\begin{array}{l}\text { Expected } \\
\text { value }\end{array}$ \\
\hline $\begin{array}{l}\text { Plasmodium_knowlesi_strain_H | product = hypothetical } \\
\text { protein, conserved in Apicomplexan s }\end{array}$ & psu|PKH_030860 & K15 & 8.42 & 57570 & $16 \%$ & 37 & 8 & 1.1 \\
\hline $\begin{array}{l}\text { Plasmodium_knowlesi_strain_H | product = conserved } \\
\text { Plasmodium protein, unknown function }\end{array}$ & psu|PKH_050250 & K16 & 9.20 & 52407 & $1 \%$ & 26 & 2 & 13 \\
\hline $\begin{array}{l}\text { Plasmodium_knowlesi_strain_H | product = dynein light } \\
\text { chain 1, putative } \mid \text { location = Pk_stra }\end{array}$ & psu|PKH_050850 & K17 & 8.75 & 23061 & $34 \%$ & 34 & 7 & 2.1 \\
\hline $\begin{array}{l}\text { Plasmodium_knowlesi_strain_H } \mid \text { product }=\text { conserved } \\
\text { Plasmodium protein, unknown function | }\end{array}$ & psu|PKH_093010 & K23 & 5.08 & 32237 & $34 \%$ & 46 & 10 & 0.13 \\
\hline $\begin{array}{l}\text { Plasmodium_knowlesi_strain_H | product }=\text { translation } \\
\text { intiation factor IF-2 | location = Pk_s }\end{array}$ & psu|PKH_050380 & K24 & 8.77 & 137165 & $5 \%$ & 28 & 12 & 7.7 \\
\hline $\begin{array}{l}\text { Plasmodium_knowlesi_strain_H } \mid \text { product }=\text { conserved } \\
\text { Plasmodium protein }\end{array}$ & PKH_050250 & K26 & 9.20 & 52407 & $1 \%$ & 29 & 2 & 6.9 \\
\hline $\begin{array}{l}\text { Plasmodium_knowlesi_strain_H } \mid \text { product }=\text { conserved } \\
\text { Plasmodium protein, unknown function }\end{array}$ & psu|PKH_091010 & $\mathrm{K} 28$ & 8.32 & 20628 & $8 \%$ & 38 & 3 & 0.82 \\
\hline $\begin{array}{l}\text { Plasmodium_knowlesi_strain_H } \mid \text { product }=\text { conserved } \\
\text { Plasmodium protein, unknown function }\end{array}$ & psu|PKH_050250 & K29 & 9.20 & 52407 & $1 \%$ & 15 & 2 & $1.6 \mathrm{e}^{+002}$ \\
\hline $\begin{array}{l}\text { Plasmodium_knowlesi_strain_H } \mid \text { product }=\text { conserved } \\
\text { Plasmodium protein, unknown function }\end{array}$ & psu|PKH_091010 & K30 & 8.32 & 20628 & $8 \%$ & 32 & 3 & 8.32 \\
\hline $\begin{array}{l}\text { Plasmodium_knowlesi_strain_H | product }=\mathrm{SOH} 1 \\
\text { homologue, putative } \mid \text { location }=\text { Pk_strainH_ch }\end{array}$ & psu|PKH_126910 & K32 & 6.90 & 16303 & $20 \%$ & 42 & 9 & 0.37 \\
\hline $\begin{array}{l}\text { Plasmodium_knowlesi_strain_H | product = hypothetical } \\
\text { protein, conserved in Apicomplexan s }\end{array}$ & psu|PKH_031640 & K33 & 9.43 & 9570 & $24 \%$ & 25 & 3 & 18 \\
\hline $\begin{array}{l}\text { Plasmodium_knowlesi_strain_H | product = } \\
\text { dihydrolipoamide dehydrogenase, putative | ocate }\end{array}$ & psu|PKH_051660 & K37 & 9.21 & 74131 & $1 \%$ & 16 & 2 & $1.2 \mathrm{e}^{+002}$ \\
\hline $\begin{array}{l}\text { Plasmodium_knowlesi_strain_H | product = conserved } \\
\text { Plasmodium protein, unknown function }\end{array}$ & psu|PKH_050250 & K38 & 9.20 & 52407 & $1 \%$ & 31 & 2 & 4.4 \\
\hline
\end{tabular}



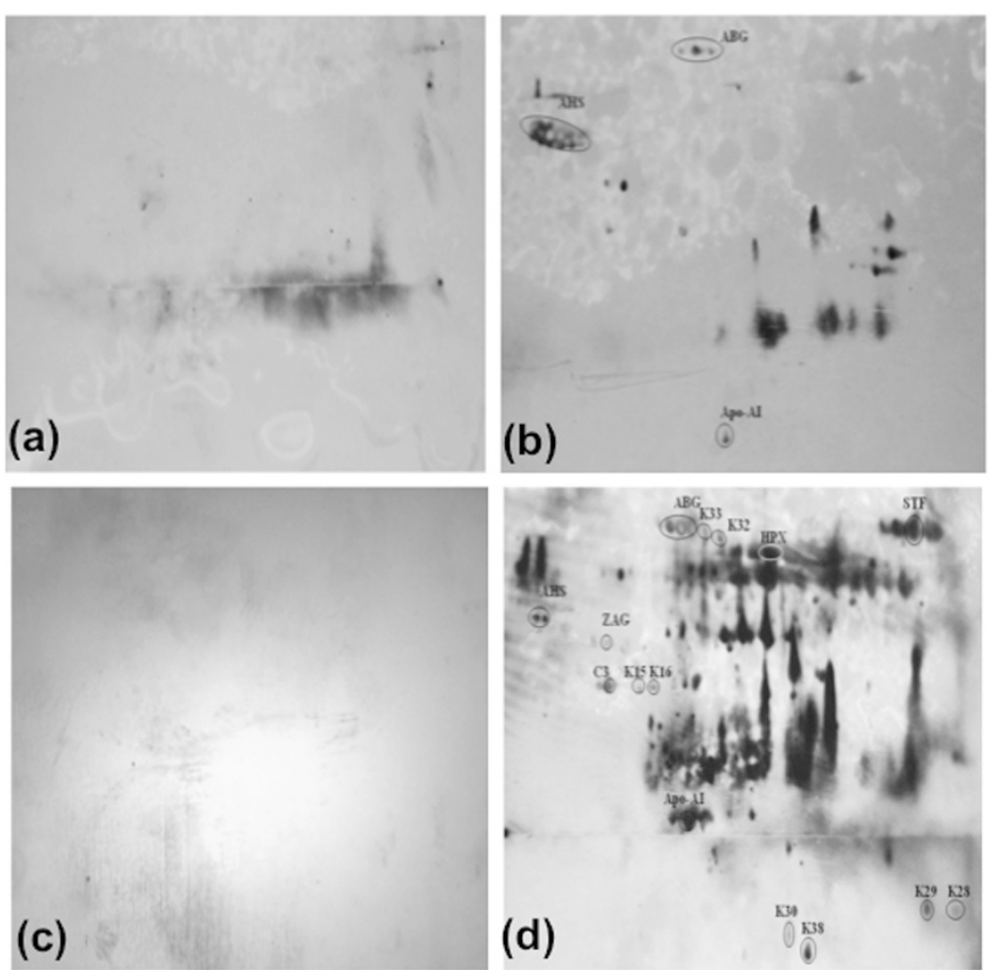

Figure 2 Western blot results following 2-DE. (a) normal pooled sera probed with normal pooled sera, (b) normal pooled sera probed with P. knowlesi pooled sera, (c) P. knowlesi pooled sera probed with normal pooled sera, (d) P. knowlesi pooled sera probed with P. knowlesi pooled sera, Unfractionated, pooled serum samples from patients and normal controls were subjected to 2-DE, transferred onto nitrocellulose membranes, and probed with pooled sera (as primary antibody) followed by monoclonal anti-human IgM-HRP (as secondary antibody).

(Figure 4). Indeed, most of the host-specific proteins display an established association with the Apo-A1-dominated network. Indeed, 10 protein partners (ABCA1, APOA2, APOB, APOC3, CFH, F2, FGA, FGB, LCAT, and TFRC) were predicted to be part of this functional interaction.

\section{Discussion}

The global impact of malaria has sparked interest in developing effective diagnostic strategies, which are not only essential for resource-limited areas where malaria is a substantial societal burden, but also for developed countries $[18,20]$. In theory, the detection of malaria parasites or antigens in patient blood should be simple. However, diagnostic efficacy is subject to many important variables, including the various stages of erythrocytic schizogony, species endemicity, interrelationship between levels of transmission, population movement, parasitemia, immunity, and species-specific symptoms [11]. Among the Plasmodium species, detection of P. knowlesi is the most challenging due to morphological similarities and physical co-localization of genetic loci on the same chromosome within an individual or species. In this respect, Carlton et al. was the first to generate and compare a synteny map of $P$. falciparum, $P$. vivax, P. knowlesi, and the rodent malaria parasites (P. yoelii,
P. berghei, and P. chabaudi) [19]. Overall, high synteny was observed between $P$. vivax and $P$. knowlesi chromosomes, with the exception of microsyntenic breaks in species-specific genes (e.g., P. knowlesi kir and SICAvar) [21]. These breaks were recognized as foci involved in the evolution of host-parasite interaction genes [22].

Proteomic analysis by 2-DE has been widely used to identify specific bacterial or viral antigens during vaccine and/or serodiagnostic test development [23]. Nevertheless, there have been few proteomic studies focused at elucidating pathogenic mechanisms or identifying potential diagnostic markers in malaria [24-26]. In the present study, we have analysed serum samples from infected individuals to identify and characterize novel markers of malaria infection using 2-DE coupled with immunoblotting techniques and mass spectrometry analysis. It is known that the immune response is relative with the presence of parasite density. Therefore, the immunoproteomics approach used in this study can be applied to identify antigens targeted by the immune system that response to infection. In addition, several studies have also reported on the protective role of $\operatorname{IgM}$ in the immune response during malaria infection $[27,28]$. Thus, both the host proteins and P. knowlesi antigens, represents the potential 


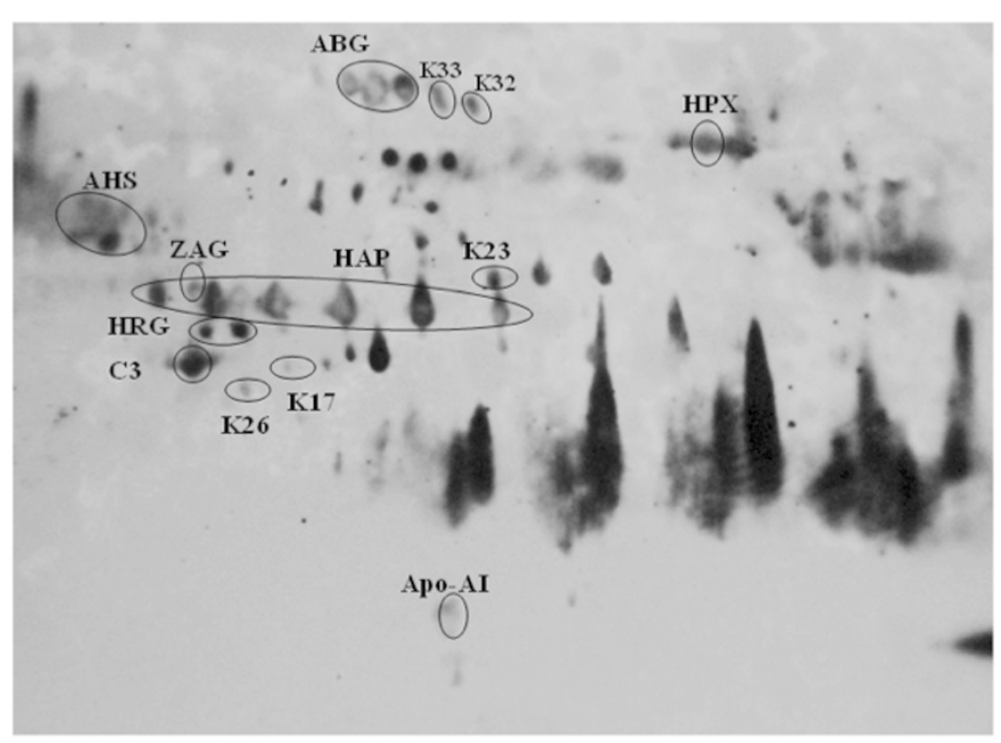

Figure 3 Western blot of $P$. knowlesi pooled sera probed with $P$. vivax pooled sera. Unfractionated, pooled serum samples from patient is subjected to 2-DE, transferred onto nitrocellulose membranes, and probed with pooled sera (as primary antibody) followed by monoclonal anti-human IgM-HRP (as secondary antibody).

candidate biomarkers that could be used in the development of future diagnostic tools for $P$. knowlesi.

It has been suggested that invasion of erythrocytes by malaria parasites involves specific interactions between parasite receptors and erythrocyte ligands [29,30]. For this process, glycoproteins on the parasite receptors bind to hydrophobic peptides on the surface of human erythrocytes. Thus, hydrophobic peptides, which can be found in the form of sialic acid-rich regions, could make cells susceptible to infection by creating a negative charge at the surface [29]. Based on our findings, TF, HPX and HAP constitute very selective biomarkers for malaria infection. These three proteins are known as iron-binding glycoproteins which are involved in the

Table 6 Functional enrichment classification of identified host-specific proteins using DAVID bioinformatics tool

\begin{tabular}{|c|c|c|c|c|c|}
\hline & & Enrichment score $^{+}$ & Protein count (\%) & Protein ID & $P$ value \\
\hline \multirow[t]{6}{*}{ Biological process } & $\begin{array}{l}\text { Regulation of response to external } \\
\text { stimulus }\end{array}$ & 2.3 & $3(27.27)$ & AHSG, C3, HRG & $4.69 \mathrm{e}-3$ \\
\hline & Cellular iron ion homeostasis & 2.13 & $3(27.27)$ & HAP, HPX, TF & $1.81 \mathrm{e}-4$ \\
\hline & Iron ion homeostasis & 2.13 & $3(27.27)$ & HAP, HPX, TF & $2.45 e-4$ \\
\hline & $\begin{array}{l}\text { Cellular di-, tri-valent inorganic cation } \\
\text { homeostasis }\end{array}$ & 2.13 & $3(27.27)$ & HAP, HPX, TF & $9.34 \mathrm{e} .3$ \\
\hline & $\begin{array}{l}\text { Di-, tri-valent inorganic cation } \\
\text { homeostasis }\end{array}$ & 2.13 & $3(27.27)$ & HAP, HPX, TF & $1.03 e-2$ \\
\hline & Cellular cation homeostasis & 2.13 & $3(27.27)$ & HAP, HPX,TF & $1.16 \mathrm{e}-2$ \\
\hline \multirow[t]{3}{*}{ Molecular function } & Endopeptidase inhibitor activity & 2.3 & $3(27.27)$ & AHSG,C3, HRG & $3.32 \mathrm{e}-3$ \\
\hline & Peptidase inhibitor activity & 2.3 & $3(27.27)$ & $\mathrm{AHSG}, \mathrm{C} 3, \mathrm{HRG}$ & $3.69 e-3$ \\
\hline & Enzyme inhibitor activity & 2.3 & $3(27.27)$ & AHSG, C3, HRG & $1.11 \mathrm{e}-2$ \\
\hline \multirow[t]{5}{*}{ Cellular component } & Extracellular region & 8.11 & $11(100)$ & $\begin{array}{l}\text { A1BG, AHSG, APOA1, AZGP1, C3, } \\
\text { FGG, HAP, HPX, HRG, LRG1, TF }\end{array}$ & $2.66 \mathrm{e}-11$ \\
\hline & Secretory granule & 2.31 & $4(36.36)$ & APOA1, FGG, HRG, TF & $3.06 \mathrm{e}-4$ \\
\hline & $\begin{array}{l}\text { Cytoplasmic membrane-bounded } \\
\text { vesicle }\end{array}$ & 2.31 & $4(36.36)$ & APOA1, FGG, HRG, TF & $7.58 \mathrm{e}-3$ \\
\hline & Membrane-bounded vesicle & 2.31 & $4(36.36)$ & APOA1, FGG, HRG, TF & $8.29 \mathrm{e}-3$ \\
\hline & Cytoplasmic vesicle & 2.31 & $4(36.36)$ & APOA1, FGG, HRG, TF & $1.16 e-2$ \\
\hline
\end{tabular}

"The classification stringency was set to high. 


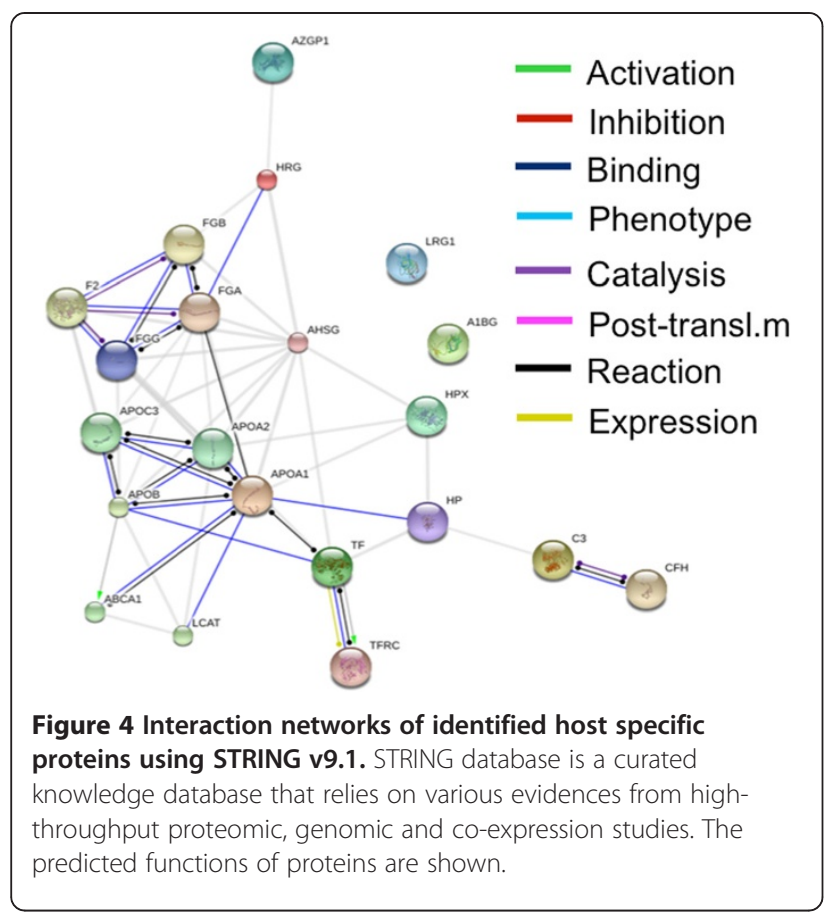

regulation of iron homeostasis that plays a key role in the innate immune response [31].

Iron is essential for the development of malaria parasite. It is known that Plasmodium parasites synthesize their own TF receptors, which can become localized on the surface of infected cells [32-34]. The delivery of extracellular iron from TF to infected erythrocytes is the source of ferric ions for malaria parasites. The alteration of TF level may influence the balance between inhibiting and promoting the survival of malaria parasite [35]. Thus, it is possible that $P$. knowlesi and $P$. vivax directly secrete TF to gain access to iron through receptormediated endocytosis.

Notably, it was reported that iron deficiency induces TF receptor expression and doubles the number of HPX surface receptors (subsequently increasing HPXmediated heme uptake in vitro) [36]. Under homeostasis, HPX can scavenge most of the free heme to form heme-HPX complex which prevent the onset of malaria [37]. Interestingly, some bacterial species, such as Haemophilus influenzae, Campylobacter jejuni, and Yersinia pestis [38-41], possess specialized iron acquisition systems for survival in hosts and are capable of heme uptake through heme-HPX complexes. Although the heme uptake activity is not parasite specific, it is possible that a similar survival system might be utilized by Plasmodium species. The expression of HPX has also been identified in several malaria studies, where this protein provides the support line of defence against haemoglobinmediated oxidative damage during intravascular haemolysis [30,42].
Additionally, our results have demonstrated that HAP is downregulated in malaria patients. However, HAP was only antigenic in sera from $P$. vivax-infected individuals and not from $P$. knowlesi-infected patients. High HAP expression can reduce symptoms associated with malaria by causing toxicity to Plasmodium parasites [43] and by removing free haemoglobin $(\mathrm{Hb})$ following Plasmodiumtriggered haemolysis. In this regard, evidence has indicated that a higher peak of parasitemia and/or parasite burden was found in P. berghei (ANKA)- or Plasmodium chabaudi-infected mice compared to that of wild type [44]. In short, decreased expression of HAP could contribute to the life-threatening levels of parasitemia observed in $P$. knowlesi-infected patients.

\section{Conclusions}

In summary, we have demonstrated the application of immunoproteomics approach to understand the immune response and identify potential candidate biomarkers for knowlesi malaria infection. Taken together, we have specifically identified TF, HPX and HAP as antigenic markers in P. knowlesi. A further investigation on the functional roles of the identified potential biomarkers in larger clinical samples will be valuable to enhance our current understanding of $P$. knowlesi and to develop effective diagnostic tools to detect knowlesi malaria.

\section{Abbreviations}

DNA: Deoxyribonucleic acid; pLDH: Plasmodium lactate dehydrogenase; 2-DE: Two-dimensional electrophoresis; SDS: Sodium dodecyl sulphate; CBB: Coomassie brilliant blue; MS: Mass spectrometry; CHCA: Cyano-4hydroxy-cinamic acid; TFA: Trifluoroacetic acid; ACN: Acetonitrile; PMF: Peptide mass fingerprinting; TBST: Tris-buffered saline-Tween 20; HRP: Horseradish peroxidase; SD: Standard deviation; PCR: Polymerase chain reaction; AHSG: Alpha-2-HS glycoprotein; TF: Serotransferrin; C3: Complement C3c; HPX: Hemopexin; ZAG1: Zinc-2-alpha glycoprotein; Apo-A1: Apolipoprotein A1; HAP: Haptoglobin; A1BG: Alpha-1-B-glycoprotein; pl: Isoelectric point; Mr: Molecular mass; FGG: Fibrinogen y chain; HO-1: Heme oxygenase-1.

\section{Competing interests}

The authors declare that they have no competing interests.

\section{Authors' contributions}

YC carried out the two-dimensional electrophoresis; mass spectrometry experiments and drafted the manuscript. CKC participated in the database search. JPK carried out the immunoassays. YLL participated in the image and the statistical analysis. Y-LW and SCBG participated in the functional analysis of identified proteins. All authors read and approved the final manuscript.

\section{Acknowledgements}

Y. Chen was supported by the following grants: UM-MoHE HIR UM.C/625/1/ HIR/MOHE/MED/16/5; E-Science Fund (12-02-03-2093) and High Impact Research MoE Grant UM.C/625/1/HIR/MOE/DENT/09.

\section{Author details}

'Department of Oral Biology \& Biomedical Sciences, Faculty of Dentistry, University of Malaya, 50603 Kuala Lumpur, Malaysia. ${ }^{2}$ Oral Cancer Research and Coordinating Center, Faculty of Dentistry, University of Malaya, Kuala Lumpur, Malaysia. ${ }^{3}$ Institute for Research in Molecular Medicine, Universiti Sains Malaysia, Gelugor, Penang, Malaysia. ${ }^{4}$ Department of Parasitology, Faculty of Medicine, University of Malaya, Kuala Lumpur, Malaysia. ${ }^{5}$ Institute of Nano Electronic Engineering (INEE), \& School of Bioprocess Engineering, Universiti Malaysia Perlis, Kangar, Perlis, Malaysia. 
Received: 8 September 2014 Accepted: 27 January 2015 Published online: 06 February 2015

\section{References}

1. Jongwutiwes S, Putaporntip C, Iwasaki T, Sata T, Kanbara H. Naturally acquired Plasmodium knowlesi malaria in human. Thailand Emerg Infect Dis. 2004;10(12):2211-3.

2. White NJ. Plasmodium knowlesi: the fifth human malaria parasite. Clin Infect Dis. 2008;46(2):172-3

3. Singh B, Daneshvar C. Human infections and detection of Plasmodium knowlesi. Clin Microbiol Rev. 2013;26(2):165-84

4. Singh B, Kim Sung L, Matusop A, Radhakrishnan A, Shamsul SS, Cox-Singh J, et al. A large focus of naturally acquired Plasmodium knowlesi infections in human beings. Lancet. 2004;363(9414):1017-24.

5. Cox-Singh J, Davis TM, Lee KS, Shamsul SS, Matusop A, Ratnam S, et al. Plasmodium knowlesi malaria in humans is widely distributed and potentially life threatening. Clin Infect Dis. 2008;46(2):165-71.

6. Daneshvar C, Davis TM, Cox-Singh J, Rafa'ee MZ, Zakaria SK, Divis PC, et al. Clinical and laboratory features of human Plasmodium knowlesi infection. Clin Infect Dis. 2009;49(6):852-60.

7. Lee KS, Cox-Singh J, Singh B. Morphological features and differential counts of Plasmodium knowlesi parasites in naturally acquired human infections. Malar J. 2009;8:73.

8. McCutchan TF, Piper RC, Makler MT. Use of malaria rapid diagnostic test to identify Plasmodium knowlesi infection. Emerg Infect Dis. 2008;14(11):1750-2.

9. Schaecher K, Kumar S, Yadava A, Vahey M, Ockenhouse CF. Genome-wide expression profiling in malaria infection reveals transcriptional changes associated with lethal and nonlethal outcomes. Infect Immun. 2005;73(9):6091-100

10. Lovegrove FE, Tangpukdee N, Opoka RO, Lafferty El, Rajwans N, Hawkes $M$, et al. Serum angiopoietin-1 and -2 levels discriminate cerebral malaria from uncomplicated malaria and predict clinical outcome in African children. PLoS One. 2009:4(3):e4912.

11. Lau YL, Fong MY, Mahmud R, Chang PY, Palaeya V, Cheong FW, et al. Specific, sensitive and rapid detection of human plasmodium knowles infection by loop-mediated isothermal amplification (LAMP) in blood samples. Malar J. 2011;10:197.

12. Chen Y, Lim BK, Peh SC, Abdul-Rahman PS, Hashim OH. Profiling of serum and tissue high abundance acute-phase proteins of patients with epithelial and germ line ovarian carcinoma. Proc Natl Acad Sci U S A. 2008;6:20.

13. Heukeshoven J, Dernick R. Improved silver staining procedure for fast staining in PhastSystem Development Unit. I. Staining of sodium dodecyl sulfate gels. Electrophoresis. 1988;9(1):28-32.

14. Shevchenko A, Wilm M, Vorm O, Mann M. Mass spectrometric sequencing of proteins silver-stained polyacrylamide gels. Anal Chem. 1996;68(5):850-8

15. da Huang W, Sherman BT, Lempicki RA. Systematic and integrative analysis of large gene lists using DAVID bioinformatics resources. Nat Protoc. 2009;4(1):44-57.

16. da Huang W, Sherman BT, Lempicki RA. Bioinformatics enrichment tools: paths toward the comprehensive functional analysis of large gene lists. Nucleic Acids Res. 2009:37(1):1-13.

17. Franceschini A, Szklarczyk D, Frankild S, Kuhn M, Simonovic M, Roth A, et al. STRING v9.1: protein-protein interaction networks, with increased coverage and integration. Nucleic Acids Res. 2013;41(Database issue):D808-15.

18. Carlton JM, Escalante AA, Neafsey D, Volkman SK. Comparative evolutionary genomics of human malaria parasites. Trends Parasitol. 2008;24(12):545-50.

19. Bell DR, Jorgensen $P$, Christophel EM, Palmer KL. Malaria risk: estimation of the malaria burden. Nature. 2005;437(7056):E3-4. discussion E-5.

20. Reyburn H, Mbakilwa H, Mwangi R, Mwerinde O, Olomi R, Drakeley C, et al. Rapid diagnostic tests compared with malaria microscopy for guiding outpatient treatment of febrile illness in Tanzania: randomised trial. BMJ. 2007:334(7590):403.

21. Pain A, Bohme U, Berry AE, Mungall K, Finn RD, Jackson AP, et al. The genome of the simian and human malaria parasite Plasmodium knowlesi. Nature. 2008;455(7214):799-803.

22. Kooij TW, Carlton JM, Bidwell SL, Hall N, Ramesar J, Janse CJ, et al. A Plasmodium whole-genome synteny map: indels and synteny breakpoints as foci for species-specific genes. PLoS Pathog. 2005;1(4):e44.

23. Yeng C, Osman E, Mohamed Z, Noordin R. Detection of immunogenic parasite and host-specific proteins in the sera of active and chronic individuals infected with Toxoplasma gondii. Electrophoresis. 2010;31(23-24):3843-9.
24. Nischik N, Schade B, Dytnerska K, Dlugonska H, Reichmann G, Fischer HG. Attenuation of mouse-virulent Toxoplasma gondii parasites is associated with a decrease in interleukin-12-inducing tachyzoite activity and reduced expression of actin, catalase and excretory proteins. Microbes Infect. 2001;3(9):689-99.

25. Gastens MH, Fischer HG. Toxoplasma gondii eukaryotic translation initiation factor 4A associated with tachyzoite virulence is down-regulated in the bradyzoite stage. Int J Parasitol. 2002;32(10):1225-34.

26. Neudeck A, Stachelhaus S, Nischik N, Striepen B, Reichmann G, Fischer $\mathrm{HG}$. Expression variance, biochemical and immunological properties of Toxoplasma gondii dense granule protein GRA7. Microbes Infect. 2002;4(6):581-90.

27. Couper KN, Phillips RS, Brombacher F, Alexander J. Parasite-specific IgM plays a significant role in the protective immune response to asexual erythrocytic stage Plasmodium chabaudi AS infection. Parasite Immunol. 2005;27(5):171-80.

28. Mustafa B, Jenneby M, Esther KA, Hastings OS, Michael GM. Immunity to Plasmodium knowlesi $\mathrm{H}$ strain malaria in olive baboons. arXiv preprint arXiv:12013232. 2012.

29. Mitchell GH, Hadley TJ, McGinniss MH, Klotz FW, Miller LH. Invasion of erythrocytes by Plasmodium falciparum malaria parasites: evidence for receptor heterogeneity and two receptors. Blood. 1986;67(5):1519-21.

30. Ray S, Renu D, Srivastava R, Gollapalli K, Taur S, Jhaveri T, et al. Proteomic investigation of falciparum and vivax malaria for identification of surrogate protein markers. PLoS One. 2012;7(8):e41751.

31. Johnson EE, Wessling-Resnick M. Iron metabolism and the innate immune response to infection. Microbes Infect. 2012;14(3):207-16.

32. Lodish HF, Small B. Membrane proteins synthesized by rabbit reticulocytes. J Cell Biol. 1975;65(1):51-64.

33. Fritsch G, Treumer J, Spira DT, Jung A. Plasmodium vinckei: suppression of mouse infections with desferrioxamine B. Exp Parasitol. 1985;60(2):171-4

34. Clark MA, Goheen MM, Cerami C. Influence of host iron status on Plasmodium falciparum infection. Front Pharmacol. 2014;5:84.

35. Delanghe JR, Langlois MR. Hemopexin: a review of biological aspects and the role in laboratory medicine. Clin Chim Acta. 2001;312(1-2):13-23.

36. Ferreira A, Balla J, Jeney V, Balla G, Soares MP. A central role for free heme in the pathogenesis of severe malaria: the missing link? J Mol Med. 2008;86(10):1097-111.

37. Rodriguez $\mathrm{MH}$, Jungery M. A protein on Plasmodium falciparum-infected erythrocytes functions as a transferrin receptor. Nature. 1986;324(6095):388-91.

38. Whitby PW, Sim KE, Morton DJ, Patel JA, Stull TL. Transcription of genes encoding iron and heme acquisition proteins of Haemophilus influenzae during acute otitis media. Infect Immun. 1997;65(11):4696-700.

39. Sikkema DJ, Brubaker RR. Outer membrane peptides of Yersinia pestis mediating siderophore-independent assimilation of iron. Biol Met. 1989;2(3):174-84

40. Cope LD, Thomas SE, Hrkal Z, Hansen EJ. Binding of heme-hemopexin complexes by soluble HxuA protein allows utilization of this complexed heme by Haemophilus influenzae. Infect Immun. 1998;66(9):4511-6.

41. Pickett $\mathrm{CL}$, Auffenberg T, Pesci EC, Sheen VL, Jusuf SS. Iron acquisition and hemolysin production by Campylobacter jejuni. Infect Immun. 1992;60(9):3872-7.

42. Cunnington AJ, Njie M, Correa S, Takem EN, Riley EM, Walther M. Prolonged neutrophil dysfunction after Plasmodium falciparum malaria is related to hemolysis and heme oxygenase-1 induction. J Immunol. 2012;189(11):5336-46.

43. Imrie H, Fowkes FJ, Michon P, Tavul L, Hume JC, Piper KP, et al. Haptoglobin levels are associated with haptoglobin genotype and alpha +-Thalassemia in a malaria-endemic area. Am J Trop Med Hyg. 2006;74(6):965-71.

44. Hunt NH, Driussi C, Sai-Kiang L. Haptoglobin and malaria. Redox Rep. 2001:6(6):389-92 